\title{
UNIDADES GENÉTICAS PARA LA CONSERVACIÓN DE Mammillaria (CACTACEAE)
}

\author{
GENETIC UNITS FOR THE CONSERVATION OF Mammillaria (CACTACEAE)
}

\author{
Sofía Solórzano*, Oswaldo Téllez, Ricardo Álvarez-Espino y Patricia Dávila
}

\begin{abstract}
Unidad de Biotecnología y Prototipos (UBIPRO), Facultad de Estudios Superiores Iztacala, Universidad Nacional Autónoma de México. Avenida de los Barrios 1. 54090, Los Reyes Iztacala, Tlalnepantla de Baz, Estado de México, México.

*Autor para correspondencia (solorzanols@unam-mx)
\end{abstract}

\section{RESUMEN}

Mammillaria agrupa a 200 especies, 164 registradas en México, 85 $\%$ son endémicas y 113 están en la Norma Oficial Mexicana NOM-059 SEMARNAT-2010. A pesar de su importancia en la biodiversidad y de su grave crisis de conservación, la escasa información documentada en estas especies no permite proponer estrategias para protegerlas. Nuestro objetivo fue estimar los niveles de diversidad genética poblacional en $M$. hernandezii, $M$. kraehenbuehlii y $M$. napina para identificar unidades genéticas de conservación. Se genotiparon con 10 loci de microsatélites 24 individuos de dos poblaciones de $M$. hernandezii y 120 individuos de cinco poblaciones para cada una de las otras dos especies. En las tres especies se estimaron niveles de heterocigosidad observada menores que los esperados ( $M$. hernandezii $0.58,0.65 ;$ M. kraehenbuehlii 0.61, 0.73; y M. napina $0.56,0.74$ ), la diversidad alélica varió de cinco ( $M$. hernandezii) a ocho alelos en las otras dos especies. Las tres especies mostraron una deficiencia de heterocigotos que puede deberse a deriva genética porque sus poblaciones son pequeñas, aunque la autofecundación también podría participar. Entre las poblaciones de las tres especies los niveles de flujo génico fueron altos, lo que indica que podría ser la deriva genética y el sistema de dispersión de polen y semillas lo que determina la estructura genética. Para $M$. kraehenbuehlii y $M$. napina se proponen tres grupos genéticos para que sean considerados como referencias para programas de conservación de estas especies y de sus hábitats. Las principales amenazas para las tres especies son la severa transformación del paisaje que aisla a sus poblaciones y el saqueo. En M. hernandezii se debe incrementar el número de poblaciones estudiadas para tener resultados concluyentes de su diversidad genética poblacional, por el momento el patrón de distribución geográfica en parches pequeños indica una severa fragmentación que insta a tomar medidas urgentes para su protección y manejo.

Palabras clave: Mammillaria hernandezii, Mammillaria kraehenbuehlii, Mammillaria napina, diversidad genética, unidades de conservación.

\section{SUMMARY}

Mammillaria groups 200 species. Mexico harbors 164 species, $85 \%$ are endemic and 113 are listed in the Official Mexican Standard NOM-059 SEMARNAT-2010. In spite of their importance in biodiversity and serious conservation crisis, the scarce information documented for these species does not allow proposals for protection strategies. Our objective was to estimate the population genetic diversity levels of $M$. hernandezii, $M$. kraehenbuehlii and $M$. napina to identify genetic conservation units. Twenty-four individuals from two $M$. hernandezii populations and 120 individuals from five populations for the other two species were genotyped with ten microsatellite loci. In the three species the observed heterozygosity levels were lower than the expected ones (M. hernandezii 0.58, 0.65; M. kraehenbuehlii 0.61, 0.73; and $M$. napina 0.56, $0.74)$. The allelic diversity ranged from five ( $M$. hernandezii) to eight alleles in the other two species. The three species showed heterozygotic deficiency possibly due to genetic drift since their populations are small, but selfing may also be involved. Gene flow levels were high for the three especies indicating that genetic drift, pollen and seed dispersal systems determine the genetic structure. Three genetic groups are proposed for $M$. kraehenbuehlii and $M$. napina to be considered as reference for conservation programs of the species and their habitats. The main threats for the three species are severe landscape transformation, which isolates the populations, and looting. For $M$. hernandezii, the number of studied populations should increase to guarantee conclusive results of its population genetic diversity. Currently, the geographic distribution pattern in small patches indicates severe habitat fragmentation that urges to take actions for its protection and management.

Index words: Mammillaria hernandezii, Mammillaria kraehenbuehlii, Mammillaria napina, genetic diversity, conservation units.

\section{INTRODUCCIÓN}

La familia Cactaceae es prácticamente de distribución exclusiva del continente americano y se reconocen de 1600 (Gibson y Nobel, 1986) a 1922 especies (Novoa et al., 2015). México se ha propuesto centro de origen de Cactaceae ya que cerca de 850 especies se registran en este país y 671 de ellas son de carácter endémico (Arias et al., 1997). En particular, para el género Mammillaria se reconocen 171 especies (Novoa et al., 2015). Este género se distribuye desde el sureste de Canadá hasta América del Sur (Speirs, 1982). Sin embargo, México concentra 82 \% de estas cactáceas y $85 \%$ son exclusivas al país (Arias et al., 1997).

El género Mammillaria agrupa a especies de talla pequeña (< 5 a $30 \mathrm{~cm}$ de altura) que pueden tener formas globosas aplanadas o tubulares, sus tallos no tienen costillas y están compuestos de tubérculos cónicos, cilíndricos, piramidales o redondos que reciben el nombre de mamilas; en sus aréolas se forman las flores y los frutos (Arias et al., 1997). Los individuos pueden tener crecimiento solitario, o ramificarse poco, o muy abundantemente formando clones de crecimiento cespitoso. Las especies de MammiIlaria producen flores de colores llamativos que pueden ser grandes o pequeñas, por lo que estas plantas son saqueadas para ser usadas como ornamento (Glass, 1998). 
En Cactaceae el saqueo de plantas y de semillas disminuyen el tamaño y el reclutamiento poblacional (GodínezÁlvarez et al., 2003). Las otras amenazas son la severa transformación y la pérdida de sus hábitats naturales que han colocado a estas especies de Mammillaria en una grave crisis de conservación. El género Mammillaria está representado a nivel mundial con 168 especies en la Lista Roja de Especies Amenazadas de la Unión Internacional para la Conservación de la Naturaleza (IUCN, http://www. iucnredlist.org, por sus siglas en inglés) de las cuales 48 $\%$ se distribuyen en México (IUCN, 2015). Actualmente, en México la NOM-059-SEMARNAT-2010 incluye a 113 especies de Mammillaria en alguna categoría de riesgo, por lo que es el género de Cactaceae más representado (DOF, 2010). Por su parte la Convención Internacional para el Comercio de Especies en Peligro de Flora y Fauna (CITES, https://cites.org/eng/app/index.php, por sus siglas en inglés), incluye en su Apéndice II a todas las especies de Mammillaria con excepción de M. pectinifera y M. solisioides que están en el Apéndice I (CITES, 2015).

Los estudios ecológicos han estimado para algunas especies de Mammillaria tasas con valores de cero en su germinación y en su reclutamiento; en consecuencia, eventualmente el tamaño de las poblaciones decrece (Contreras y Valverde, 2002; Valverde y Zavala-Hurtado, 2006). En contraste, para otras especies como M. supertexta (Avendaño-Calvo et al., 2013) y M. gaumeri (Ferrer et al., 2011) se estima un crecimiento en sus poblaciones. Las densidades poblacionales en Mammillaria varían de < 1 individuo (e.g. M. gaumeri; Ferrer et al., 2011) hasta 9 $\mathrm{m}^{-2}$ (e.g. M. solisioides, Macías-Arrastio; com. pers. ${ }^{1}$ ). Los individuos suelen encontrarse en agregados que ocupan pequeñas áreas y las poblaciones se distribuyen en parches discontinuos (Ferrer et al., 2011; Peters y Martorell, 2001; Solórzano y Dávila, 2015; Téllez et al., 2015; Valverde y Zavala-Hurtado, 2006). También se tienen contribuciones sobre sus comportamientos demográficos (e.g. Ferrer et al., 2011; Peters y Martorell, 2001; Valverde y ZavalaHurtado, 2006), atributos de su historia de vida que permiten entender abundancia (Leirana-Alcocer y Parra Tabla, 1999) y otros han determinado el estado de conservación desde una perspectiva ecológica para algunas especies (Contreras y Valverde, 2002; Peters et al., 2014; Valverde y Zavala-Hurtado, 2006).

Actualmente, se cuenta con información de la genética poblacional para siete especies de Mammillaria (Solórzano et al., 2016) que presentan distinta extensión de distribución geográfica, modo de crecimiento (clonal/solitario) y diferencias en sus densidades poblacionales. Estos estudios mostraron una amplia variación en los niveles de

'Fabián F. Macías-Arrastio. Estudiante de maestría en la FES Iztacala, UNAM. diversidad y de estructura genética. También concluyeron que los tamaños y el aislamiento geográfico de las poblaciones son los dos factores ecológicos que afectan la diversidad; en tanto que la estructura genética está influenciada por los procesos de deriva génica y de flujo génico. En las especies sin autofecundación los niveles altos de endogamia indican que las poblaciones tienen tamaños efectivos pequeños (Frankham et al., 2002), lo que es posible de considerar para Mammillaria debido a los niveles tan altos de endogamia que se han documentado (Solórzano et al., 2016), aunque se debe determinar el sistema reproductivo de las especies bajo estudio.

Para cuatro especies de Mammillaria se han identificado unidades genéticas con fines de conservación. Las unidades genéticas son grupos de individuos de una población o grupos de poblaciones que se distinguen ya sea por su alta riqueza alélica/genotípica, o por tener alelos/haplotipos/genotipos que no se presentan en otras poblaciones. Estas unidades se propusieron con la finalidad de identificar prioridades de conservación intraespecíficas, ya que es común que no se puedan conservar en su totalidad las poblaciones de una especie (Ryder, 1986). En Mammillaria las unidades genéticas se han identificado a partir de los niveles de estructura genética y diversidad genética poblacional (Solórzano et al., 2014; Solórzano et al., 2016), y en M. crucigera se sumó la información de estructura demográfica (Solórzano y Dávila, 2015).

Para distinguir a estas unidades, Moritz (1994) recomendó el uso de marcadores moleculares, los que deben detectar el polimorfismo suficiente para que puedan separar a los individuos y a las poblaciones. Además, este autor precisó que los marcadores moleculares que se basan en polimorfismos homólogos identifican grupos genéticos que reflejan una historia evolutiva y que se conocen como ESUs ("evolutionary significant units"). En contraste, si se usa un marcador molecular sujeto a la homoplasia entonces estas unidades se reconocen como MUs ("management units") y éstas enfatizan una distribución no aleatoria de la diversidad genética dentro y entre poblaciones sin importar los procesos evolutivos que las moldearon (Moritz, 1994).

De estos dos tipos de unidades genéticas para conservación se ha aplicado el de MUs para tres especies de Mammillaria con marcadores microsatélites (Solórzano et al., 2014; Solórzano y Dávila, 2015); sin embargo, no se han identificado ESUs debido a que no se han encontrado suficientes polimorfismos en regiones de cloroplasto. Las MUs identificadas para Mammillaria han permitido identificar poblaciones que son prioridades de conservación, así como inferir los procesos genético-evolutivos subyacentes. Por lo anterior, en el presente estudio se evaluó la 
diversidad genética poblacional de M. hernandezii, M. kraenhuenbuelii y M. napina con la finalidad de identificar unidades genéticas para su conservación.

Un estudio reciente analizó la distribución geográfica en detalle de las tres especies aquí estudiadas (Figura 1) (Téllez et al., 2015). Este estudio estimó su extensión geográfica total (EOO, por sus siglas en inglés) y su área de ocupación real (AOO, por sus siglas en inglés) con sistemas de información geográfica. La EOO mide la distribución total y se calcula como el área total de un polígono que incluye a todas las localidades registradas para una especie. En tanto que la AOO se calcula a la menor escala geográfica posible para cuantificar el área realmente ocupada por los individuos (Gaston y Fuller, 2009). Para las tres especies aquí estudiadas se describió una distribución geográfica en pequeños parches con distinto grado de aislamiento (Téllez et al., 2015). Además, estas tres especies son endémicas de México y están en las listas de la NOM-059-SEMARNAT-2010 (DOF, 2010) y de la IUCN (Arias et al., 2013;
Arias y Martorell, 2013; Arias y Zavala-Hurtado, 2013). En este contexto, se espera para M. hernandezii, M. kraenhuenbuelii y M. napina encontrar niveles bajos de diversidad genética, tanto de heterocigosidad como de diversidad alélica y una marcada estructura poblacional que permita distinguir grupos genéticos.

\section{MATERIALES Y MÉTODOS}

\section{Especies de estudio}

Mammillaria hernandezii. Se registra en pequeños lomeríos calizos ubicados en elevaciones de $2000 \mathrm{~m}$ en el Valle de Tehuacán-Cuicatlán, sus hábitats son suelos calizos desnudos inmersos en pastizal de Cynodon dactylon y Aristida adscensionis (Téllez et al., 2015). En total se registran nueve poblaciones para esta especie (Peters y Martorell, 2001), en las que el número de individuos por metro cuadrado varia de dos a siete. Estas poblaciones se incluyen en un polígono de EOO de 16 km²; sin embargo, el área realmente

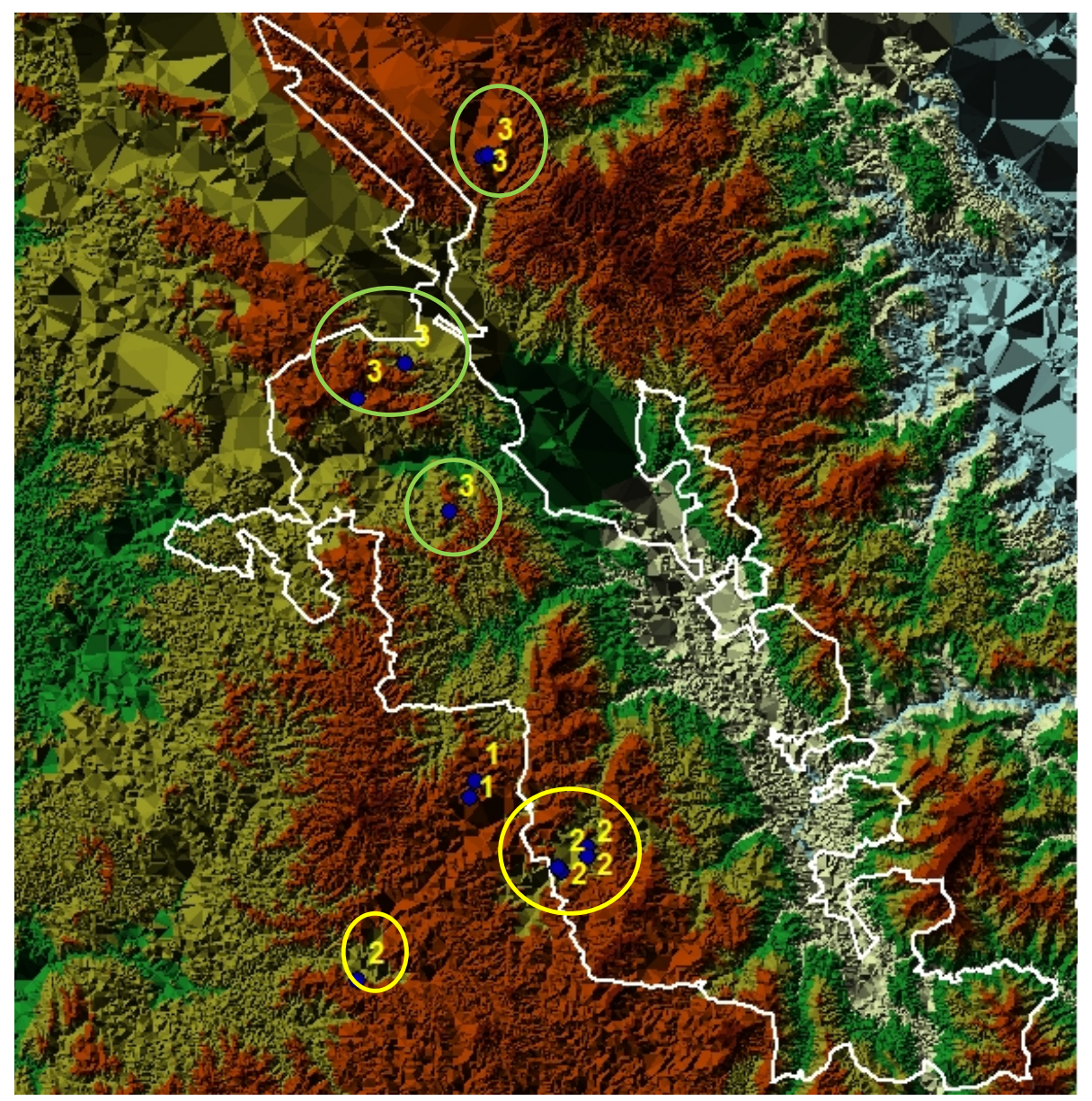

Figura 1. Ubicación de las localidades de estudio en el área Tehuacán-Cuicatlán para las tres especies. $M$ hernandezii (1), M. kraehenbuehlii (2) y M. napina (3). Los círculos encierran a las poblaciones de las Unidades de Conservación identificadas para M. kraehenbuehlii (amarillo) y M. napina (verdes). 
ocupada (AOO) por las poblaciones suma $2.5 \mathrm{~km}^{2}$ (Téllez et al., 2015). Los individuos son de crecimiento solitario y alcanzan de 2 a $3.5 \mathrm{~cm}$ de diámetro y hasta $1.5 \mathrm{~cm}$ de altura. Esta especie florece de octubre a diciembre y los frutos se presentan entre diciembre y enero (Arias et al., 1997). La NOM-059-SEMARNAT-2010 (DOF, 2010) incluye a esta especie en la categoría de protección especial, la lista de la IUCN la clasifica en peligro de extinción (Arias y Martorell, 2013) y CITES la incluye en el Apéndice II (CITES, 2015).

Mammillaria kraehenbuehlii. En total esta especie registra 13 poblaciones aisladas entre sí, 12 de ellas se ubican dentro del Valle de Tehuacán-Cuicatlán y solo una fuera del Valle en la región de la Mixteca Alta (Peters y Martorell, 2001; Téllez et al., 2015). Esta especie ramifica densamente por lo que el crecimiento es cespitoso y un solo individuo puede cubrir hasta $3 \mathrm{~m}^{2}$, sus densidades poblacionales varían de 22 a 85 individuos $/ 500 \mathrm{~m}^{2}$. A esta especie se le encuentra en elevaciones de 700 a $2500 \mathrm{~m}$ donde se desarrolla matorral xerófito en el que dominan Dasylirion serratifolium y Ephedra compacta. Las poblaciones más alejadas están separadas por distancias superiores a 50 km, por lo que el polígono de extensión total cubre 1823 $\mathrm{km}^{2}$, pero el área de ocupación real de las poblaciones suma $8.23 \mathrm{~km}^{2}$ (Téllez et al., 2015). Las ramas son cortas y cilíndricas de 3 a $12 \mathrm{~cm}$ de altura con 2.5 a $3.5 \mathrm{~cm}$ de diámetro y esta especie florece de marzo a junio (Arias et al., 1997), y en junio se registran los últimos frutos (Téllez et al., 2015). La NOM-059-SEMARNAT-2010 (DOF, 2010) incluye a esta especie en la categoría de protección especial, la lista de la IUCN la clasifica en la categoría de preocupación menor (Arias y Zavala-Hurtado, 2013) y el CITES la incluye en el Apéndice II (CITES, 2015).

Mammillaria napina. De las 13 poblaciones donde se registra esta especie, cinco se ubican fuera del Valle de Tehuacán-Cuicatlán y las ocho poblaciones restantes dentro de este Valle, que ocupan elevaciones de 1800 a 2100 m. Los individuos más grandes tienen alturas de $6 \mathrm{~cm}$ y diámetros de $3 \mathrm{~cm}$, son de crecimiento solitario y se registran densidades de 1 a 3 individuos $\mathrm{m}^{-2}$. En sus hábitats destacan los cardonales de Pseudomitrocereus fulviceps o de izotales de Yucca periculosa. La extensión total del polígono que encierra a las 13 poblaciones cubre $739 \mathrm{~km}^{2}$, que contrasta con los 5 km² del área real de ocupación (Téllez et al., 2015). Esta especie florece entre marzo y mayo, los frutos con semillas maduras se registran de septiembre a octubre y producen de 35 a 42 semillas por fruto, cuya dispersión no se ha documentado (Téllez et al., 2015), pero se conoce que los frutos son serotinos y sus semillas son viables hasta 8 años después de que se formaron (Rodríguez-Ortega et al., 2006). La NOM-059-SEMARNAT-2010 (DOF, 2010) incluye a esta especie en la categoría de amenazada, la lista de la IUCN la clasifica en la categoría de críticamente en peligro
(Arias et al., 2013) y ClTES la incluye en el Apéndice II (ClTES, 2015).

\section{Análisis genéticos}

Las muestras para el análisis genético provienen de poblaciones naturales, para M. napina y M. kraehenbuehlii se colectaron muestras de aproximadamente $0.5 \mathrm{~cm}^{2}$ de tejido vegetativo del tallo de 14 a 20 individuos para cinco poblaciones de cada especie sumando 120 muestras. Para el caso de Mammillaria hernandezii solo se colectaron 24 muestras provenientes de dos poblaciones. Además, para esta última especie, por el tamaño tan pequeño de su tallo (<2 cm de altura), se decidió colectar parte de la flor (corola) en sustitución de tejido vegetativo de tallo.

A partir del tejido vegetativo del tallo o de las flores se aisló el ADN genómico total (ADNg) tras seguir el protocolo del DNA easy plant kit (Qiagen, California) y usar de 70 a $100 \mathrm{mg}$ de tejido que fue pulverizado con nitrógeno líquido. Para confirmar que se tuviera ADNg, cada muestra se cargó en geles de agarosa al 0.8 \%, teñidos con bromuro de etidio, los cuales fueron visualizados con luz UV. Para las tres especies se ensayaron ocho loci de microsatélites diseñados previamente para M. crucigera (Solórzano et al., 2009) y dos más que fueron monomórficos para la especie fuente, pero en M. huitzilopochtli y M. supertexta (Solórzano et al., 2014), M. solisioides y M. zephyranthoides (Solórzano et al., 2016) resultaron polimórficos. En total, 10 loci se amplificaron (Cuadro 1) con la técnica de reacción en cadena de la polimerasa (PCR, por sus siglas en inglés) en reacciones individuales y se establecieron para cada especie y cada locus las condiciones específicas.

El ciclo base de PCR fue de desnaturalización inicial de $94{ }^{\circ} \mathrm{C}$ durante $3 \mathrm{~min}$, y 35 ciclos que consistieron en desnaturalización $\left(94^{\circ} \mathrm{C}\right.$ por $\left.10 \mathrm{~s}\right)$, alineamiento $\left(50-62{ }^{\circ} \mathrm{C}\right.$ por 10 s), extensión $\left(72{ }^{\circ} \mathrm{C}\right.$ por $\left.10 \mathrm{~s}\right)$ y una extensión final a $72{ }^{\circ} \mathrm{C}$ por $5 \mathrm{~min}$. Las concentraciones en un volumen final de 25 $\mu \mathrm{L}$ fueron del buffer $10 \mathrm{X}(0.8 \mathrm{X})$, oligonucleótidos 5'-3' y 3'5' (0.2 pM), dNTPs (0.4 mM), MgCl (5 mM), ADN-polimerasa $(0.04 \mathrm{U})$ y $\sim 20 \mathrm{ng} \mathrm{uL}^{-1}$ de ADNg. Los oligonucleótidos marcados llevaron los fluorocromos NED o FAM (Applied Biosystem). Se comprobó la amplificación de cada locus de cada muestra en geles de agarosa al $1.2 \%$ teñidos con bromuro de etidio y visualizados con UV.

La electroforesis de la reacción de PCR con oligonucleótido marcado se realizó en el secuenciador ABI 3100 del Instituto de Biología, UNAM. Los electroferogramas de microsatélites se analizaron con el programa PeakScanner (Applied Biosystem) para obtener el tamaño de los alelos en pares de bases de cada muestra para cada locus de cada población y especie. Los análisis estadísticos se realizaron 


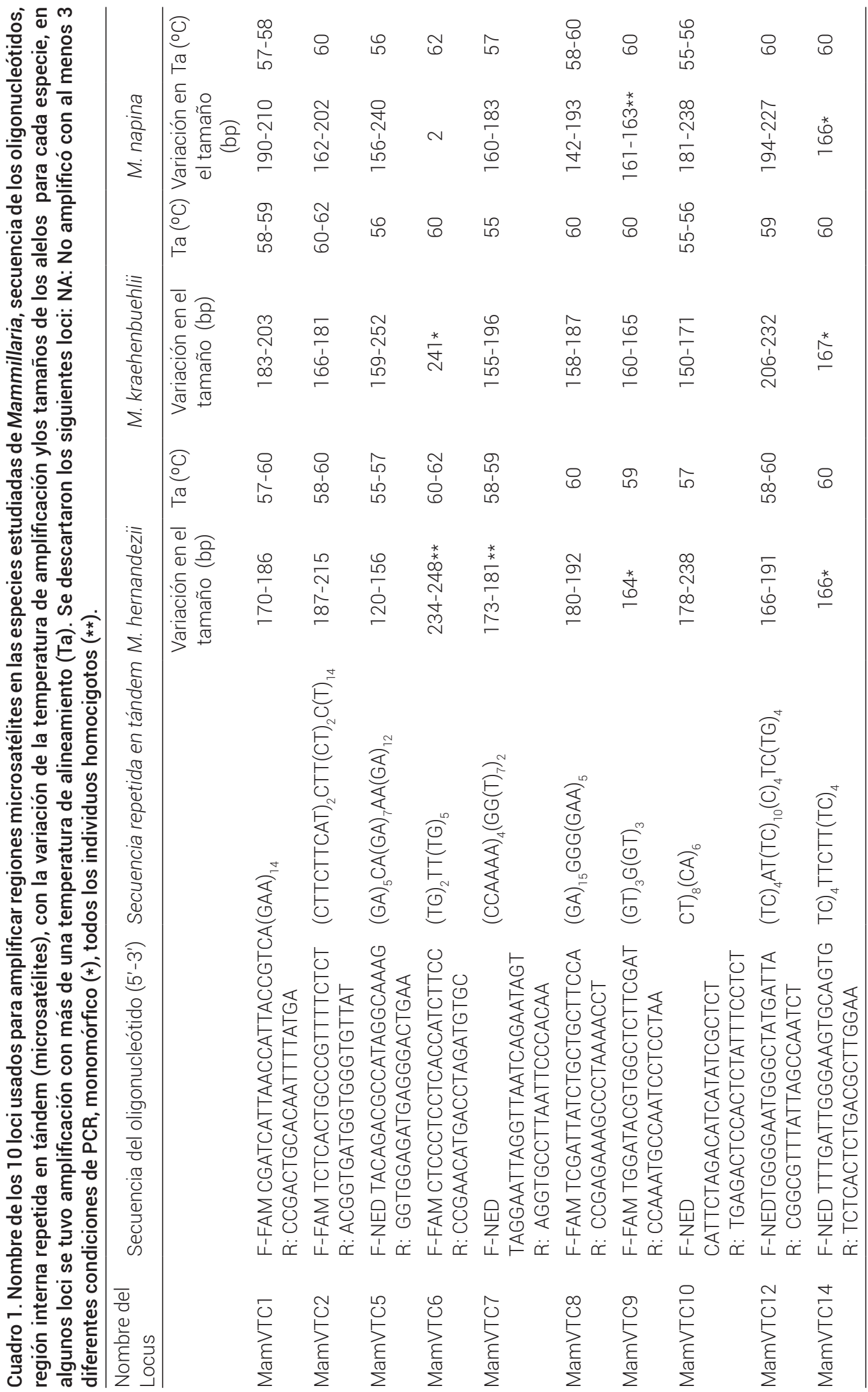


en jerarquías a nivel de locus, promedio de los loci para cada población y para datos agrupados que consideran a las poblaciones como una gran población total; para estos análisis se usó el programa Arlequin 3.0 (Excoffier et al., 2005). Con Genetic Data Analysis software (Lewis y Zaykin, 2001) se estimaron los alelos exclusivos de cada población. Los loci que resultaron polimórficos se analizaron para buscar alelos nulos con el programa MicroChecker (Van Oosterhout et al., 2004) y no se encontraron, por lo que todos los loci polimórficos se usaron en los análisis estadísticos. Los niveles de diversidad se estimaron desde la heterocigosidad observada $\left(\mathrm{H}_{0}=\right.$ número de individuos heterocigotos/total de individuos analizados; Nei, 1977) y esperada $\left(H_{F}=1-\Sigma p i^{2}\right.$ donde $p i=$ frecuencia alélica; Nei, 1977), la diversidad alélica ( $N_{A}=$ número de alelos encontrados/total de loci analizados, Frankham et al., 2002) y número efectivo de alelos $\left(N_{E F A}=1 / 7-\Sigma p i^{2}\right.$, Frankham et al., 2002). También, los indices de fijación (Wright, 1949) con el coeficiente de endogamia por locus $\left(F_{I S}=H_{E}-H o / H_{E}\right)$, el promedio de endogamia por población $\left(F_{I S}=H_{s}-H_{s} / H_{s}\right)$ y para el total de poblaciones agrupadas como una sola población $\left(F_{I T}=H_{T}-H_{,} / H_{T}\right)$.

A nivel de locus se estimó la desviación del equilibrio Hardy-Weinberg entre heterocigosidad observada y esperada para examinar la significancia con el procedimiento de Bonferroni mediante el método descrito por Sokal y Rohlf (1994). La estructura genética se determinó a partir del coeficiente de diferenciación genética $R_{\mathrm{ST}}$ ajustado para marcadores de microsatélites (Michalakis y Excoffier, 1996), con los niveles de flujo génico entre poblaciones. En particular se examinó el modelo de flujo génico de aislamiento por distancia con la prueba de Mantel (Liedloff, 1999). También se examinaron las hipótesis de los grupos genéticos (K) soportados con los valores de $\Phi_{\text {CT }}$ y su valor de P asociado con el programa SAMOVA 1.0 (Dupanloup et al., 2002) junto con los porcentajes de variación molecular (AMOVA) sin agrupamientos a priori. Las distancias genéticas de Nei entre poblaciones se calcularon con Arlequin 3.0 (Excoffier et al., 2005) y se graficaron en MEGA 6.0 (Tamura et al., 2013).

\section{RESULTADOS}

Las tres especies de estudio mostraron niveles relativamente altos de heterocigosidad; sin embargo, los valores observados fueron menores a los valores que se esperaron en equilibrio Hardy-Weinberg (Cuadro 2), en consecuencia, los niveles de endogamia $\left(F_{I T}\right)$ fueron altos en cada una de las tres especies. El número promedio de alelos que contribuyeron significativamente $\left(\mathrm{N}_{\mathrm{EfA}}\right)$ con los niveles de heterocigosidad fueron menos de dos, lo que indica que el resto de los alelos por sus frecuencias $<0.05$ no influyeron en los niveles de heterocigosidad, por tanto el promedio de alelos por locus $\left(\mathrm{N}_{A}\right)$ fue superior a cinco en las tres especies estudiadas.

M. hernandezii. Las dos poblaciones estudiadas mostraron variación en diversidad genética. El Gavilán mostró niveles más altos de heterocigosidad y sin niveles significativos de endogamia (Cuadro 3). Loma de Biznaga fue la población en donde se estimaron niveles altos de endogamia, diversidad alélica y número de alelos exclusivos. Entre estas dos poblaciones se estimaron niveles altos de flujo génico $(\mathrm{Nm}=5)$ y la diferenciación genética no fue significativa $\left(R_{S T}=0.08, P=0.08\right)$.

M. kraehenbuehlii. La heterocigosidad observada en las cinco poblaciones fue menor que la esperada (Cuadro 3) y aunque el promedio del coeficiente de endogamia para las poblaciones no fue significativo $\left(F_{\mid S}=0.14, P=0.5\right)$, sí fue significativo cuando se agruparon los datos de las cinco poblaciones para ser analizados como una sola población (Cuadro 2). A nivel de población, solo dos (Tequixtepec 1 y 3) no mostraron niveles significativos de endogamia, pero las restantes tres sí los tuvieron (Cuadro 3). En todas las poblaciones se identificaron alelos exclusivos que variaron de cuatro (Tamaluzulapan) a 13 (Tequixtepec 2).

La diferenciación genética promedio entre las cinco poblaciones fue alta y significativa $\left(R_{S T}=0.26, P=0.02\right)$ al igual que los niveles de flujo génico $(\mathrm{Nm}=3)$. Las comparaciones pareadas entre poblaciones mostraron que Tamazulapan, la población más alejada geográficamente del resto, fue la que presentó niveles más altos de diferenciación genética $(0.05$ a 0.48) y a la vez la que presentó los niveles más bajos de flujo génico $(<1)$ con las demás poblaciones (Figura 2); sin embargo, los niveles de flujo génico no se correlacionaron con la distancia geográfica por lo que se descartó el modelo de aislamiento por distancia $\left(R^{2}=0.28, P=0.65\right)$ para esta especie.

De las tres hipótesis para examinar grupos genéticos ninguna se soportó estadísticamente y el de $K=3$, fue marginalmente significativa pero tuvo el mismo valor de $\Phi_{\text {Ст }}$ que $K=2$ (Cuadro 4). La variación genética dentro de las poblaciones fue la que contribuyó con el mayor porcentaje seguida de la que se cuantificó entre grupos. El análisis fenético mostró que Tamazulapan tiene la mayor distancia genética, además es la más alejada geográficamente del resto y aunque Tequixtepec 1 y 3 se organizaron en el mismo nodo no son las más cercanas geográficamente (Figura 3a).

M. napina. La heterocigosidad observada en las cinco poblaciones fue menor que la esperada (Cuadro 3) y el promedio del coeficiente de endogamia para las poblaciones fue alto y significativo $\left(F_{I S}=0.28, P=0.0002\right)$, al igual que 
Cuadro 2. Diversidad genética para las especies de estudio a partir del muestreo poblacional. El número de individuos y de poblaciones se presentan en paréntesis. Los niveles de heterocigosidad esperados $\left(H_{E}\right)$ son más altos que los observados $\left(\mathrm{H}_{\mathrm{O}}\right)$, el número efectivo de alelos $\left(\mathrm{N}_{\mathrm{EFA}}\right)$ está por debajo que el promedio de alelos por locus $\left(\mathrm{N}_{\mathrm{A}}\right)$, los niveles de endogamia $\left(F_{I T}\right)$ son significativos $(P<0.05)$ para las tres especies después del ajuste de Bonferroni.

\begin{tabular}{llllll}
\hline Especie/estimador & $\mathrm{H}_{\mathrm{E}}$ & $\mathrm{H}_{\mathrm{O}}$ & $\mathrm{N}_{\mathrm{EfA}}$ & $\mathrm{N}_{\mathrm{A}}$ & $\mathrm{F}_{\text {IT }}(\mathrm{P})$ \\
\hline M. hernandezii (24:2) & 0.65 & 0.58 & 1.53 & 5.2 & $0.21(0.002)$ \\
M. kraehenbuehlii (120:5) & 0.73 & 0.61 & 1.4 & 7 & $0.40(0.01)$ \\
M. napina (120:5) & 0.74 & 0.56 & 1.35 & 8 & $0.38(0.00)$ \\
\hline
\end{tabular}

Cuadro 3. Diversidad genética para cada población analizada en las tres especies de estudio.

\begin{tabular}{llccccl}
\hline Especie & Población/Estimador & $\mathrm{H}_{\mathrm{E}}$ & $\mathrm{H}_{0}$ & $\mathrm{~N}_{\mathrm{A}}$ & $\mathrm{N}_{\text {AEx }}$ & $\mathrm{F}_{\text {IS }}$ \\
\hline M. hernandezii & El Gavilán & 0.61 & 0.57 & 4.5 & 8 & $0.06(0.18)$ \\
M. kraehenbuehlii & Loma de Biznaga & 0.70 & 0.46 & 5.2 & 10 & $0.14 *(0.001)$ \\
& San Martín & 0.76 & 0.70 & 6 & 10 & $0.14 *(0.03)$ \\
& Tamazulapan & 0.65 & 0.58 & 4.2 & 4 & $0.18 *(0.01)$ \\
& Tequixtepec 1 & 0.69 & 0.63 & 5 & 10 & $0.06(0.62)$ \\
M. napina & Tequixtepec 2 & 0.72 & 0.49 & 7 & 13 & $0.32 *(0.03)$ \\
& Tequixtepec 3 & 0.75 & 0.68 & 7.5 & 9 & $0.09(0.20)$ \\
& Morelos I & 0.62 & 0.49 & 8.5 & 8 & $0.09(0.96)$ \\
& Morelos II & 0.73 & 0.53 & 6.5 & 6 & $0.19(0.11)$ \\
& Nopala & 0.66 & 0.43 & 4.2 & 7 & $0.59(0.001)$ \\
& Teloxtoc & 0.77 & 0.65 & 9.7 & 18 & $0.28(0.03)$ \\
& Xochiltepec & 0.76 & 0.61 & 6.7 & 6 & $0.47(0.001)$ \\
\hline
\end{tabular}

$\mathrm{H}_{\mathrm{E}}$ : heterocigosidad esperada; $\mathrm{H}_{0}$ : heterocigosidad observada; $\mathrm{N}_{\mathrm{A}}$ : diversidad alélica; $\mathrm{N}_{\mathrm{AEX}}$ : número exclusivo de alelos; $\mathrm{F}_{I S}$ : coeficiente de endogamia dentro de la población; el asterisco indica $\mathrm{P}<0.05$.

cuando se agruparon los datos de las cinco poblaciones que representan a una sola población (Cuadro 2). Cada una de las cinco poblaciones mostró niveles significativos de endogamia (Cuadro 3). En todas las poblaciones se identificaron alelos exclusivos que varían de seis (Morelos II) a 18 (Teloxtoc), en promedio las poblaciones tuvieron una diversidad alélica cercana a siete alelos por locus.

La diferenciación genética promedio entre las cinco poblaciones fue alta y significativa $\left(R_{S T}=0.19, P=0.003\right)$ y el flujo génico promedio que se estimó entre todas las poblaciones fue alto $(\mathrm{Nm}=4.2)$. Las comparaciones pareadas entre poblaciones mostraron que Nopala, la población más alejada geográficamente del resto, es la que presentó los niveles más altos de diferenciación genética (0.17 a 0.38) con el resto de las poblaciones y a la vez los niveles de flujo génico más bajos $(<1)$ con las demás poblaciones (Figura 2). El modelo de aislamiento por distancia confirmó que en esta especie los niveles de flujo génico se correlacionaron con la distancia geográfica, así que las poblaciones más alejadas geográficamente tuvieron entre sí los niveles más bajos de flujo génico $\left(R^{2}=-0.55, P=0.01\right)$.
En $M$. napina, la hipótesis de $K=3$ grupos genéticos se sustentó estadísticamente $(P<0.05)$ aunque el valor de $\Phi_{\text {CT }}$ fue más alto en la hipótesis de $K=2$ no se soportó estadísticamente $(P=0.28$, Cuadro 5$)$. Los tres grupos genéticos mostraron una concordancia con la ubicación geográfica de las poblaciones. La variación dentro de las poblaciones se identificó como la principal fuente de variación en la especie, seguida de la que se cuantificó entre grupos. La población de Nopala se separa también del resto en el análisis fenético y además es la que tiene la mayor distancia genética (Figura 3b).

\section{DISCUSIÓN}

Los resultados de diversidad genética aquí presentados son similares a los descritos en otras especies de Mammillaria (Solórzano et al., 2014; Solórzano y Dávila, 2015) y otras cactáceas pequeñas como Astrophytum asterias (Terry et al., 2012). En estas especies de cactáceas pequeñas se han encontrado valores relativamente altos de heterocigosidad pero valores bajos de diversidad alélica. Esta relación de alta heterocigosidad y baja diversidad alélica 


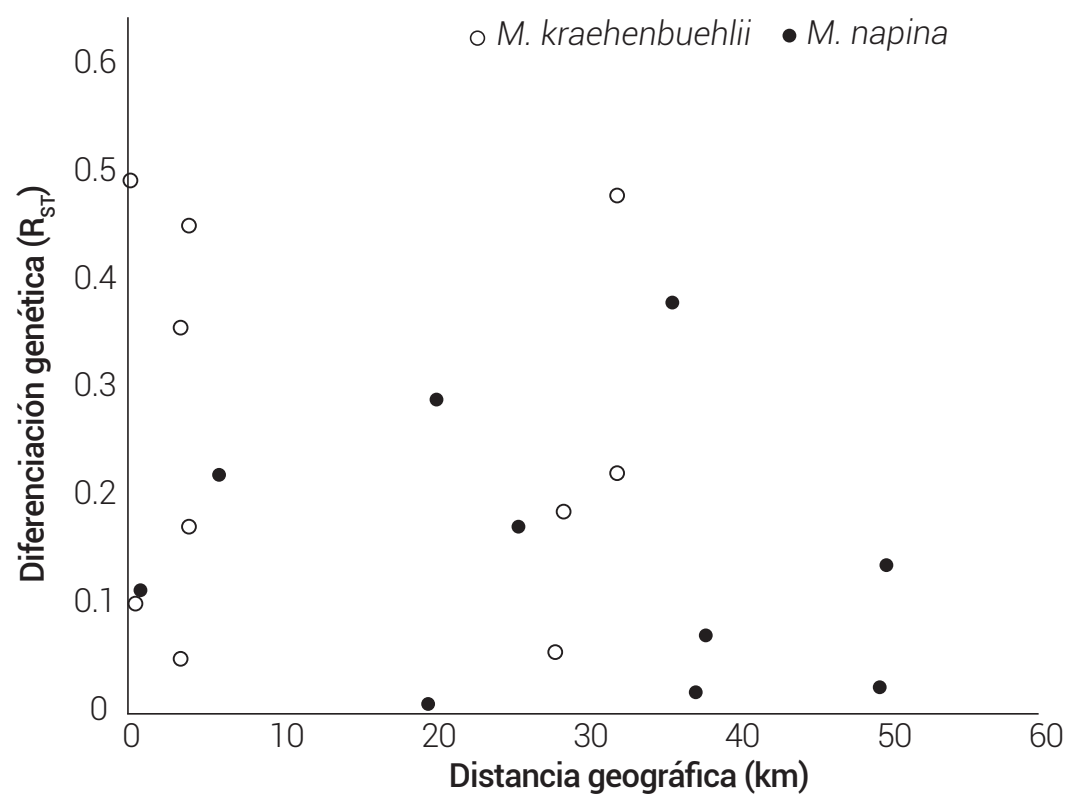

Figura 2. Relación entre la distancia geográfica $(\mathrm{km})$ y los niveles de flujo génico $(\mathrm{Nm})$ entre poblaciones de $M$. kraehenbuehlii y M. napina, sólo esta última se ajustó al modelo de aislamiento por distancia.

Cuadro 4. Hipótesis de grupos genéticos para $M$. kraehenbuelii se presentan número de grupos y su composición con los porcentajes de variación entre tres jerarquías y del valor de $\Phi_{C T}$ y el valor de significancia.

\begin{tabular}{|c|c|c|c|c|}
\hline $\begin{array}{l}\text { Hipótesis examinada con } \\
\text { número de grupos }(K)\end{array}$ & Entre grupos & $\begin{array}{l}\text { Entre poblaciones dentro } \\
\text { de los grupos }\end{array}$ & Dentro de poblaciones & $\Phi_{\text {CT }}(P)$ \\
\hline $\begin{array}{l}\mathbf{K}=\mathbf{2} \\
\text { 1: San Martín; } \\
\text { 2: Tequixtepec1, Tequixtepec3 } \\
\text { Tamazulapan, Tequixtepec2 }\end{array}$ & 27 & 11 & 62 & $0.27(0.20)$ \\
\hline $\begin{array}{l}\mathbf{K}=\mathbf{3} \\
\text { 1: San Martín; } \\
\text { 2: Tamazulapan, Tequixtepec2 } \\
\text { 3: Tequixtepec1, Tequixtepec3 }\end{array}$ & 27 & 5 & 68 & $0.27(0.07)$ \\
\hline $\begin{array}{l}\mathbf{K}=\mathbf{4} \\
\text { 1: San Martín; } \\
\text { 2: Tequixtepec1 } \\
\text { 3: Tamazulapan, Tequixtepec2 } \\
\text { 4: Tequixtepec3 }\end{array}$ & 25 & 5 & 70 & $0.25(0.10)$ \\
\hline
\end{tabular}

ha sido discutida previamente por Allendorf (1986) quien sugiere que cuando se tiene este resultado es porque en las poblaciones ocurrió un cuello de botella drástico de corta duración (< 10 generaciones).

La probabilidad de que haya ocurrido cuello de botella se ha sugerido para otras especies de Mammillaria (Solórzano et al., 2014); sin embargo, se necesitan los análisis con otros marcadores (i.e. secuencias) para poder discernir si este cuello de botella ocurrió recientemente por causas antrópicas o son de origen. En las tres especies, a pesar de que los niveles de heterocigosidad son relativamente altos, también lo son los niveles de endogamia lo que pue- de ser una consecuencia indirecta de la deriva génica que causa la pérdida de alelos en las poblaciones pequeñas, pero también pueden deberse a la autofecundación. No se cuenta con información sobre el sistema reproductivo para alguna de las tres especies, aunque en campo se observó que las tres tienen hercogamia (los estigmas están a una altura por encima de las anteras), lo que sugiere un mecanismo para evitar autofecundación.

En Mammillaria los individuos de las tallas más grandes son los que tienden a producir semillas, lo que eventualmente determina el reclutamiento en las poblaciones (e.g. Ferrer et al., 2011; Leirana-Alcocer y Parra Tabla, 1999; 

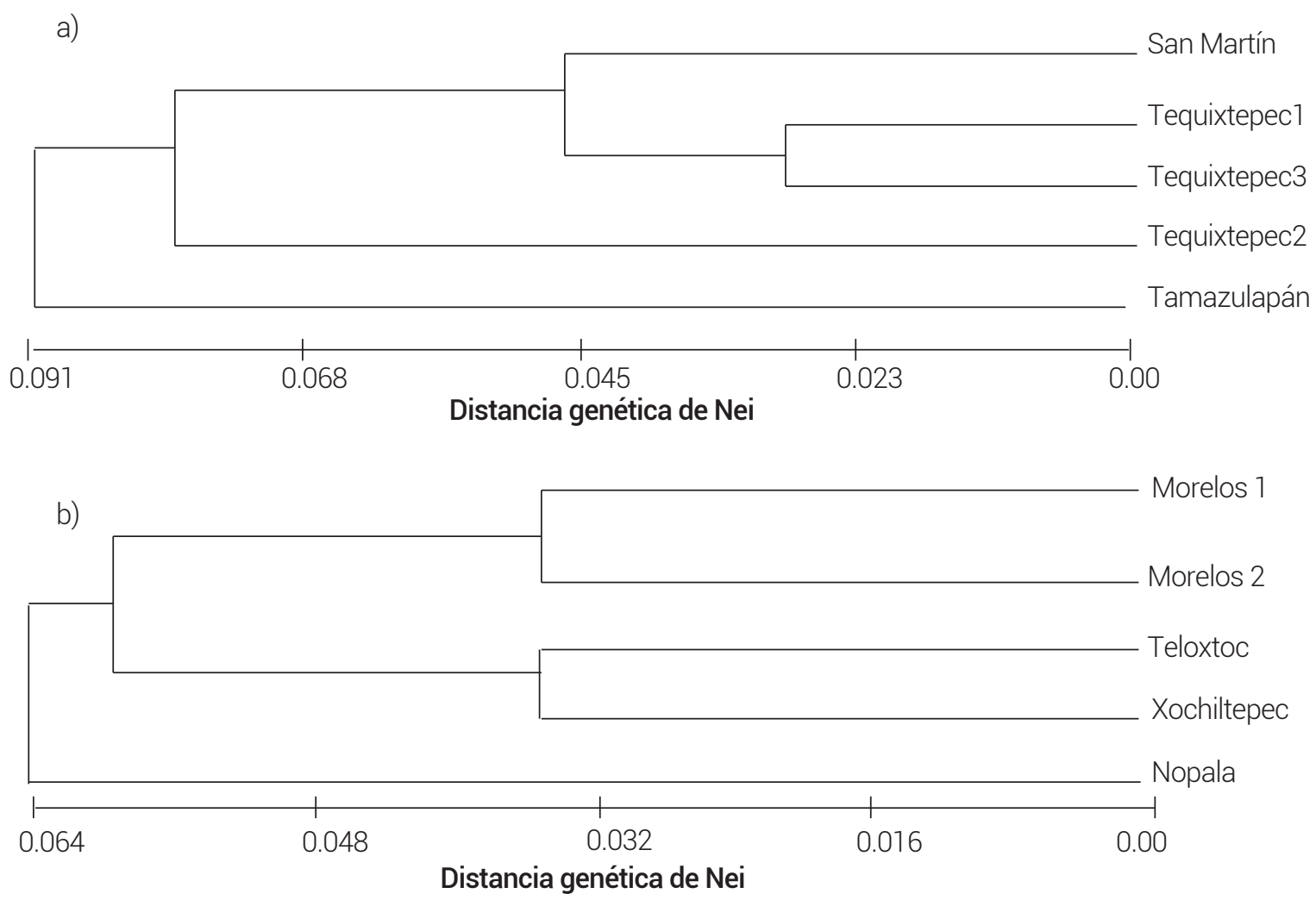

Figura 3. Relación fenética entre las poblaciones de a) M. kraehenbuehlii y de b) M. napina.

Cuadro 5. Hipótesis de grupos genéticos para $M$. napina se presentan los resultados de las hipótesis de $\mathrm{K}$ grupos genéticos y su composición con los porcentajes de variación entre tres jerarquías y del valor de $\Phi_{C T}$ y el valor de significancia $(P)$ con astérisco marcado con valor significativo.

\begin{tabular}{|c|c|c|c|c|}
\hline K grupos & Entre grupos & $\begin{array}{c}\text { Entre poblaciones } \\
\text { dentro de los grupos }\end{array}$ & $\begin{array}{c}\text { Dentro de } \\
\text { poblaciones }\end{array}$ & $\Phi_{C T}(P)$ \\
\hline $\begin{array}{l}\mathrm{K}=\mathbf{2} \\
\text { 1: Morelos II } \\
\text { 2: MorelosI, Teloxtoc, Xochiltepec, } \\
\text { Nopala }\end{array}$ & 24.7 & 0.30 & 75 & $0.25(0.28)$ \\
\hline $\begin{array}{l}\mathbf{K}=\mathbf{3} \\
\text { 1: Xochiltepec, Teloxtoc } \\
\text { 2: Morelosl, MorelosIl } \\
\text { 3: Nopala }\end{array}$ & 36 & 4 & 60 & $0.14(0.05) *$ \\
\hline $\begin{array}{l}\mathbf{K}=\mathbf{4} \\
\text { 1: Xochiltepec, } \\
\text { 2: Morelosl, MorelosIl } \\
\text { 3: Nopala } \\
\text { 4: Teloxtoc }\end{array}$ & 22 & 3 & 75 & $0.10(0.7)$ \\
\hline
\end{tabular}

Valverde y Zavala-Hurtado, 2006); sin embargo, estos individuos son también los más solicitados en el mercado ilegal (Glass, 1998; IUCN, 2015), lo que causa decremento en los tamaños poblacionales y disminución en el reclutamiento (Ferrer et al., 2011). Así que un factor crítico para mantener la diversidad genética poblacional es que se observen y se cumplan las normatividades que protejen a estas especies, para eliminar el saqueo de individuos y con ello garantizar los procesos demográficos de reproducción y reclutamiento, que eventualmente podrían incrementar los niveles de diversidad genética. 
Por otro lado, las tres especies no mostraron la misma relación entre niveles de flujo génico y la diferenciación genética, así como entre la distancia geográfica y la distancia genética. Lo anterior sugiere que en algunas especies de Mammillaria el aislamiento geográfico no es el factor que estructura genéticamente a las poblaciones, sino que es la deriva génica y potencialmente una dispersión pobre de los alelos (i.e. polen y semillas). Sin embargo, como el flujo génico es un proceso que mantine la variación alélica entre poblaciones (Frankham et al., 2002) se recomienda que se protejan los hábitats de estas especies de modo que se permita el flujo de genes dentro y entre poblaciones.

El muestreo poblacional en M. hernandezii se debe incrementar para tener resultados concluyentes sobre su diversidad genética y su distribución espacial. Por el momento, se recomienda que se considere a todas las poblaciones como prioridades para su conservación con la finalidad de vigilar la severa transformación de sus hábitats. La distribución total de esta especie está parcialmente protegida ya que cerca del $50 \%$ de sus poblaciones se ubican dentro de la reserva de la biosfera Tehuacán-Cuicatlán y el resto fuera de los límites de área natural protegida (Téllez et al., 2015). En estas últimas poblaciones se incrementa el riesgo de desaparecer por transformación del uso de suelo y por saqueo de los individuos. En el caso de M. kraehenbuelii no se sustentaron significativamente las tres hipótesis examinadas de $\mathrm{K}$ grupos genéticos $(K=2, K=3$ y $K=4)$ y los valores de $\Phi_{\text {CT }}$ tuvieron el mismo valor para los dos primeros grupos.

Por ello, para apoyar una propuesta de conservación más robusta se integró la información de la distribución geográfica de la especie. De acuerdo con esta información $M$. kraehenbuelii se distribuye solamente en el estado de Oaxaca y muestra tres áreas geográficas principales en las que se segregan sus poblaciones (Figuras 11 y 19 de Téllez et al., 2015). El área más norteña se encuentra cerca del poblado Tepelmeme Villa Morelos y las otras dos en la región de la Mixteca Alta, una de ellas cercana a Tequistepec y la otra a Villa de Tamazulapan del Progreso, esta última representa la distribución más sureña de la especie.

Esta distribución geográfica se reflejó en el agrupamiento de $K=3$ y por eso se propone que estas áreas geográficas sean la referencia para la conservación de esta especie. En el caso de M. napina tres grupos genéticos se sustentaron estadísticamente y también reflejaron la distribución geográfica de las poblaciones (Figura 14, Téllez et al., 2015) por lo que se propone que se consideren como áreas prioritarias para la conservación. Esta recomendación es importante de considerar debido a que la mayoría de las poblaciones se ubican fuera del polígono de la reserva de la biosfera Tehuacán-Cuicatlán lo que incrementa su vulnerabilidad al saqueo y a la desaparición de sus hábitats.

\section{CONCLUSIONES}

Las tres especies estudiadas, $M$. hernandezii, M. kraenhuenbuelii y M. napina, presentaron niveles de diversidad genética que sugieren que las poblaciones son pequeñas por lo que están sujetas a los efectos más severos de la deriva génica. Para respaldar la conservación de estas tres especies se propone como estrategia que se sigan los grupos genéticos identificados para que se consideren para un programa de conservación y de manejo. Las poblaciones que se ubican dentro de la reserva de la biosfera Tehuacán-Cuicatlán podrían mantenerse si se cumplen las normatividades para proteger a estas tres especies; sin embargo, las tres especies tienen poblaciones fuera de la reserva que pueden tener un mayor riesgo de desaparecer por lo que merecen más atención de las autoridades.

Las poblaciones de cada una de las especies estudiadas son afectadas de modo distinto por el aislamiento por distancia, pero las tres especies muestran un patrón insular con distinto grado de aislamiento. Este aislamiento podría tener efectos severos sobre los niveles de diversidad debido a que las poblaciones son pequeñas. Particularmente, M. hernandezii podría ser más vulnerable por tener las poblaciones más pequeñas y más fragmentadas por lo que se recomienda una atención especial a la protección de sus hábitats. Las áreas geográficas donde se ubican las poblaciones de estas tres especies, estén dentro o no de la reserva, pueden ser una referencia para dirigir un esfuerzo mayor de vigilancia de las especies y de sus hábitats.

\section{AGRADECIMIENTOS}

Los resultados aquí presentados fueron generados en los proyectos de investigación HKO4O (CONABIO), Dr. Oswaldo Téllez, responsable, y del proyecto PAPIIT-DGAPA IN217208, Dra. Sofía Solorzano, responsable. La logística para el trabajo de campo y el muestreo de las poblaciones fueron apoyados por el proyecto Banco de Semillas FesIztacala-Kew UK, Dra. Patricia Dávila, responsable. Gracias a todas las comunidades de los municipios y ejidos que permitieron el acceso para el trabajo de campo. Gracias a los técnicos de la Unidad de Síntesis del IBT-UNAM, por la síntesis de los oligonucleótidos, la electroforesis de microsatélites se realizó con el apoyo de la M. en C. Laura Márquez del IB-UNAM. 


\section{BIBLIOGRAFÍA}

Allendorf F. W. (1986) Genetic drift and the loss of alleles versus heterozygosity. Zoo Biology 5:181-190.

Arias M. S., S. Gama L. y L. U. Guzmán C. (1997) Flora del Valle de Tehuacán-Cuicatlán. Fascículo 95. CACTACEAE Juss. Instituto de Biología, UNAM. México. 146 p.

Arias S. and C. Martorell (2013) Mammillaria hernandezii. The IUCN Red List of Threatened Species. http://dx.doi.org/10.2305/IUCN UK.2013-1.RLTS.T152282A618523.en. (Mayo 2017).

Arias S., T. Valverde, A. Zavala-Hurtado and C. Martorell (2013) Mammillaria napina. The IUCN Red List of Threatened Species. http:// dx.doi.org/10.2305/IUCN.UK.2013-1.RLTS.T152476A641047. en. (Mayo 2017).

Arias S. and A. Zavala-Hurtado (2013) Mammillaria kraehenbuehlii. The IUCN Red List of Threatened Species. http://www.iucnredlist. org/details/152498/0. (Mayo 2017).

Avendaño-Calvo T., A. Flores and G. Manzanero (2013) Estudio poblacional de Mammillaria supertexta Mart. Ex Pfeiff. en el Valle de Cuicatlán, Oaxaca, México. In: Estudio en Zonas Áridas de Oaxaca: Homenaje al Dr. Alejandro Flores Martínez. M. Briones-Salas, G. I. Manzanero M. y G. González Pérez (eds.). Carteles Editores. Centro Interdisciplinario de Investigación para el Desarrollo Integral Regional, Instituto Politécnico Nacional, Unidad Oaxaca. Oaxaca, México. pp:44-56.

CITES, Convention on International Trade in Endangered Species of Wild Fauna and Flora (2015) Appendices I, II and III. https://www.cites.org/ sites/default/files/eng/app/2015/E-Appendices-2015-02-05. pdf. (Mayo 2015).

Contreras C. and T. Valverde (2002) Evaluation of the conservation status of a rare cactus (Mammillaria crucigera) through the analysis of its population dynamics. Journal of Arid Environments 51:89102.

DOF, Diario Oficial de la Federación (2010) Norma Oficial Mexicana NOM059-SEMARNAT-2010. Protección Ambiental - Especies Nativas de México de Flora y Fauna Silvestres - Categorías de Riesgo y Especificaciones para su Inclusión, Exclusión o Cambio. Lista de Especies en Riesgo. Diario Oficial de la Federación. (Segunda sección). 30 de diciembre de 2010.70 p.

Dupanloup I., S. Schneider and L. Excoffier (2002) A simulated annealing approach to define the genetic structure of populations. Molecular Ecology 11:2571-2581.

Excoffier L., G. Laval and S. Schneider (2005) ARLEQUIN Version 3.0: an integrated software package for population genetics data analysis. Evolutionary Bioinformatics Online 1:47-50. A Windows executable version Arlequin ver 3 can be freely downloaded on http://cmpg.unibe.ch/software/arlequin3.

Ferrer M., R. Durán, M. Méndez, A. Dorantes y G. Dzib (2011) Dinámica poblacional de genets y ramets de Mammillaria gaumeri cactácea endémica de Yucatán. Boletín de la Sociedad Botánica de México 89:83-105

Frankham R., J. D. Ballou and D. A. Briscoe (2002) Introduction to Conservation Genetics. Cambridge University Press. Cambridge, UK. $644 \mathrm{p}$.

Gaston K. J. and R. A. Fuller (2009) The sizes of species' geographic ranges. Journal of Applied Ecology 46:1-9.

Gibson A. C. and P. S. Nobel (1986) The Cactus Primer. Harvard University Press. Cambridge, Massachusetts. $286 \mathrm{p}$.

Godínez-Álvarez H., T. Valverde and P. Ortega-Baes (2003) Demographic trends in the Cactaceae. The Botanical Review 69:173-203.

Glass C. (1998) Guide to the Identification of Threatened Cacti of México. CONABIO-CANTE. México. 200 p.

IUCN, Red List of Threatened Species (2015) Version 2015-4. www. iucnredlist.org. (Enero 2016).

Tamura, K., G. Stecher, D. Peterson, A. Filipski and S. Kumar (2013) MEGA6: Molecular evolutionary genetics analysis version 6.0. Molecular Biology and Evolution 30:2725-2729.

Lewis P. and D. Zaykin (2001) Genetic Data Analysis V1.1. http://phylogeny.uconn.edu/software/.

Liedloff A. (1999) (Version 2.0): Mantel Nonparametric Test Calculator.
Queensland University of Technology: Brisbane, Australia.

Leirana-Alcocer J. and V. Parra-Tabla (1999) Factors affecting the distribution, abundance and seedling survival of Mammillaria gaumeri, an endemic cactus of coastal Yucatán, México. Journal of Arid Environments 41:421-428

Michalakis Y. and L. Excoffier (1996) A generic estimation of population subdivision using distances between alleles with special reference for microsatellite loci. Genetics 142:1061-1064.

Moritz C. (1994) Defining "evolutionary significant units" for conservation. Trends in Ecology and Evolution 9:373-375.

Nei M. (1977) F-statistics and analysis of gene diversity in subdivided populations. Annals of Human Genetics 41:225-233.

Novoa A., J. J. Le Roux, M. P. Robertson, J. R. U. Wilson and D. M. Richardson (2015) Introduced and invasive cactus species: a global review. AoB PLANTS 7:plu078, doi:10.1093/aobpla/plu078.

Van Oosterhout C., W. F. Hutchinson, D. P. M. Wills and P. Shipley (2004) MICRO-CHECKER: software for identifying and correcting genotyping errors in microsatellite data. Molecular Ecology Notes 4:535-538

Peters E. M., S. Arizaga, C. Martorell, R. Zaragoza and E. Ezcurra (2014) Distribución geográfica y estado de conservación de las poblaciones de Mammillaria pectinifera. Revista Mexicana de Biodiversidad 85:942-952.

Peters R. M. y C. Martorell (2001) Conocimiento y Conservación de las Mamilarias Endémicas del Valle de Tehuacán-Cuicatlán. Informe Final SNIB-CONABIO Proyecto No. R166. Universidad Nacional Autónoma de México. Instituto de Ecología. México, D. F. 38 p.

Rodríguez-Ortega C., M. Franco and M. C. Mandujano (2006) Serotiny and seed germination in three threateaned species of Mammillaria (Cactaceae). Basic and Applied Ecology 7:533-544

Ryder 0. A. (1986) Species conservation and systematics: the dilemma of subspecies. Trends in Ecology and Evolution 1:9-10

Sokal R. R. and F. J. Rohlf (1994) Biometry: The Principles and Practices of Statistics in Biological Research. 3a. Edición. W. H. Freeman \& Co (Sd). New York. $880 p$.

Solórzano S., S. Arias and P. Dávila (2016) Genetics and conservation of plant species of extremely narrow geographic range. Diversity 8:1-12.

Solórzano S. and P. Dávila (2015) Identification of conservation units of Mammillaria crucigera (Cactaceae): perspectives for the conservation of rare species. Plant Ecology \& Diversity 8:559-569.

Solórzano S., P. D. Cuevas-Alducin, V. García-Gómez and P. Dávila (2014) Genetic diversity and conservation of Mamillaria huitzilopochtli and $M$. supertexta, two threatened species endemic of the semiarid region of Central Mexico. Revista Mexicana de Biodiversidad 85:565-575.

Solórzano S., A. C. Cortés-Palomec, A. Ibarra, P. Dávila and K. Oyama (2009) Isolation, characterization and cross-amplification of polymorphic microsatellite loci in the threatened endemic Mammillaria crucigera (Cactaceae). Molecular Ecology Resources 9:156158.

Speirs D. (1982) The cacti of Western Canada: Part 3. The National Cactus and Succulent Journal 37:53-54

Téllez V. O., P. Dávila y R. X. Álvarez (2015) Evaluación de Conservación de Especies de Cactaceae en la Reserva de la Biosfera TehuacánCuicatlán. Informe Final SNIB-CONABIO Proyecto No. HKO4O. Universidad Nacional Autónoma de México. Facultad de Estudios Superiores Iztacala. México. D. F. 56 p.

Terry M. K., A. E. Pepper, A. W. Strong, D. M. Tarin, D. M. Price and J. R. Manhart (2012) Genetic structure of a population of the endangered star cactus (Astrophytum asterias) in Southern Texas. The Southwestern Naturalist 57:182-188

Valverde P. L. and J. A. Zavala-Hurtado (2006) Assessing the ecological status of Mammillaria pectinifera Weber (Cactaceae), a rare and threatened species endemic of the Tehuacán-Cuicatlán region in Central Mexico. Journal of Arid Environments 64:193208

Wright S. (1949) The genetical structure of populations. Annals of Eugenics 15:323-354. 
